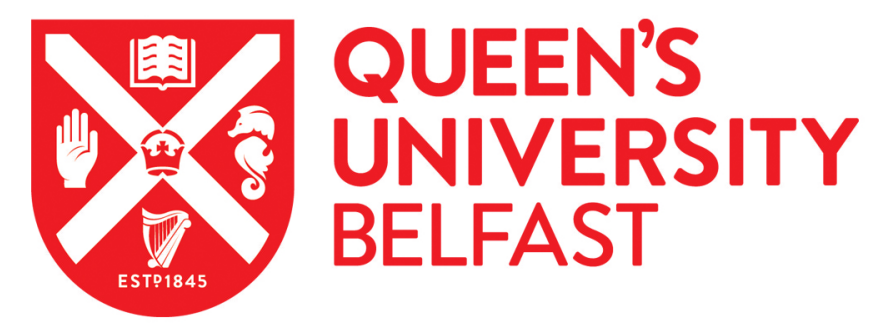

\title{
Accuracy of diagnosis at routine psychiatric assessment in patients presenting to an accident and emergency department
}

Taggart, C., O'Grady, J., Stevenson, M., Hand, E., McClelland, R., \& Kelly, C. (2006). Accuracy of diagnosis at routine psychiatric assessment in patients presenting to an accident and emergency department. Gen Hosp Psychiatry, 28(4), 330-335.

Published in:

Gen Hosp Psychiatry

Queen's University Belfast - Research Portal:

Link to publication record in Queen's University Belfast Research Portal

\section{General rights}

Copyright for the publications made accessible via the Queen's University Belfast Research Portal is retained by the author(s) and / or other copyright owners and it is a condition of accessing these publications that users recognise and abide by the legal requirements associated with these rights.

Take down policy

The Research Portal is Queen's institutional repository that provides access to Queen's research output. Every effort has been made to ensure that content in the Research Portal does not infringe any person's rights, or applicable UK laws. If you discover content in the Research Portal that you believe breaches copyright or violates any law, please contact openaccess@qub.ac.uk. 


\title{
Accuracy of diagnosis at routine psychiatric assessment in patients presenting to an accident and emergency department
}

\author{
Catherine Taggart, M.R.C.Psych. ${ }^{\mathrm{a}}$, John O'Grady, B.Sc. ${ }^{\mathrm{b}}$, Mike Stevenson, B.Sc., F.S.S ${ }^{\mathrm{c}}$, \\ Elaine Hand, M.Sc. ${ }^{\mathrm{c}}$, Roy Mc Clelland, F.R.C.Psych. ${ }^{\mathrm{b}}$, Chris Kelly, M.R.C.Psych., \\ ${ }^{a}$ Department of Psychiatry, Belfast City Hospital, Belfast, BT9 7AB, Ireland \\ ${ }^{\mathrm{b}}$ Division of Psychiatry and Neuroscience, Queen's University Belfast, Ireland \\ ${ }^{\mathrm{c}}$ Education and Research Centre, Royal Group of Hospitals Trust, Belfast, Ireland \\ Received 2 December 2005; accepted 5 May 2006
}

\begin{abstract}
Objective: This study aimed to compare diagnoses obtained from routine psychiatric assessment in accident and emergency (A\&E) settings with those from a standardized diagnostic interview.

Methods: Using Cohen's kappa, the agreement between diagnoses from routine psychiatric assessment and structured interview was calculated. Further statistical analysis was used to investigate differences between some of the main diagnostic subgroups.

Results: The overall kappa value for all diagnoses was .47. Adjustment disorder, major depressive disorder and alcohol misuse/dependence were commonly identified, both at routine assessment and on structured interview. There was a disparity between the two diagnostic systems in the extent to which major depressive disorder and adjustment disorder were identified. Further analysis between these two groups was inconclusive as to the reasons for this difference.

Conclusions: There was moderate agreement between clinical and standardized diagnoses. Anxiety disorders were poorly identified at routine assessment. There is difficulty distinguishing between adjustment disorder and depressive episode in the A\&E setting.

(C) 2006 Elsevier Inc. All rights reserved.
\end{abstract}

Keywords: Accident and emergency; Psychiatric assessment; Deliberate self-harm; Adjustment disorder; Major depressive episode

\section{Introduction}

Many patients presenting to accident and emergency (A\&E) departments require psychiatric assessment, the majority following deliberate self-harm (DSH). We sought to compare diagnoses reached at psychiatric evaluation in A\&E settings with diagnoses from a standardized diagnostic interview. We are aware of only one similar previous study [1]. The A\&E setting is not always ideal for psychiatric interview, and in addition, assessments are often constrained by time and lack of available background information. Despite this, initial assessments are a basis for decisions regarding further management and, as A\&E may be the only point of contact with a psychiatric professional, it is important that a broadly accurate working diagnosis is made. We hypothesized that in our sample of A\&E

\footnotetext{
* Corresponding author.

E-mail address: chris.kelly@bch.n-i.nhs.uk (C. Kelly).
}

presenters, there would be a preponderance of adjustment disorders diagnosed clinically but that the diagnostic instrument would diagnose major depressive disorder more readily. We also looked more specifically at certain subgroups where there was likely to be particular diagnostic difficulty or disagreement.

\section{Methods}

\subsection{Clinical and research assessment}

Diagnosis reached at routine clinical assessment of patients presenting at A\&E departments was compared with diagnosis from a structured interview. Routine assessment during normal working hours was done by a member of the liaison team (specialist registrar, liaison nurse, social worker, senior house officer or consultant psychiatrist) and by a senior house officer after working hours. All cases seen by nursing and social work staff had medical supervision. 
Most assessments were carried out in the A\&E department. Some patients required medical admission for either cardiac monitoring or acetylcysteine treatment. These assessments took place on the medical ward. They were carried out in the same time frame as those in the A\&E department.

The data used for comparison were initially collected as part of the "Ireland, North/South, Urban/Rural Epidemiology of Suicide (INSURE) Study." This is a three-phase study of suicide and suicidal behavior, which is being conducted at seven sites in Ireland over a 3-year period. Our data have been taken from Phase 2, which is a 1-year case control study of patients presenting as psychiatric emergencies. The information was collected in a large urban A\&E department between July 2001 and April 2002. Approval for the study was obtained from the Queen's University of Belfast Ethics Committee. The INSURE researcher (JO'G) was present in the A\&E department daily from Monday to Friday. On these days, every patient referred for routine psychiatric assessment was asked to take part in the study. Of these, $75 \%$ had deliberately harmed themselves or attempted suicide (a breakdown of the reasons why psychiatric assessment was requested is shown in Table 1). The researcher was not present at night, but most patients who present after working hours were routinely asked to remain for assessment the following morning and were also seen by the researcher at this time. Thus, an attempt was made to capture a sample of patients that was representative of those presenting both by day and during the night.

Consenting patients had the Structured Clinical Interview for DSM-IV (SCID) administered. We do not have a record of those who did not consent.

The SCID takes approximately $1-2 \mathrm{~h}$ to complete. The researcher who carried out the SCID interviews had attended a 3-day training program, consisting of theoretical explanation, practical demonstration and videotaped practice. Interrater reliability was assessed against a gold standard and was satisfactory.

One hundred sixty-seven patients consented to take part in the study and completed the SCID. Patients with dementia or learning disability and patients whose first language was not English were excluded. Patients who had already taken part in the study were not included again if they had any further presentations to A\&E within the study period. Most of the research interviews took place within

Table 1

Reasons for psychiatric assessment in A\&E

\begin{tabular}{lc}
\hline & $\%(n)$ \\
\hline Self-harm & $75.3(116)$ \\
Overdose & $67.5(104)$ \\
Self-laceration & $6.5(10)$ \\
Poisoning with exhaust fumes & $0.6(1)$ \\
Jumping from bridge & $0.6(1)$ \\
Concern about mental state (mood, psychotic symptoms) & $14.3(22)$ \\
Alcohol/drug misuse & $9.7(15)$ \\
Somatoform symptoms & $0.6(1)$ \\
\hline
\end{tabular}

Table 2

Comparison of diagnoses between clinical and SCID assessments

\begin{tabular}{lcc}
\hline & $\begin{array}{l}\text { Clinical } \\
\text { diagnosis, \% }(n)\end{array}$ & $\begin{array}{l}\text { SCID } \\
\text { diagnosis, \% }(n)\end{array}$ \\
\hline Bipolar disorder & $1.3(2)$ & $2.6(4)$ \\
Major depressive disorder $^{\mathrm{a}}$ & $19.5(30)$ & $36.4(56)$ \\
Psychotic disorders $^{\mathrm{b}}$ & $3.2(5)$ & $5.2(8)$ \\
Alcohol misuse/dependence & $30.5(47)$ & $20.1(31)$ \\
Drug misuse/dependence $^{\text {Substance-induced disorders }}{ }^{\mathrm{c}}$ & $3.9(6)$ & $2.6(4)$ \\
Anxiety disorders $^{\mathrm{d}}$ & $0.6(1)$ & $5.2(8)$ \\
Eating disorders $_{\text {Adjustment disorder }}$ & $1.3(2)$ & $11.7(18)$ \\
No Axis I disorder & $1.3(2)$ & $1.3(2)$ \\
\hline
\end{tabular}

${ }^{a}$ Including dysthymia and psychotic mood disorder.

${ }^{\mathrm{b}}$ Schizophrenia, schizoaffective disorder, delusional disorder and brief psychotic disorder.

${ }^{c}$ Substance-induced mood disorder and substance-induced psychotic disorder.

${ }^{\mathrm{d}}$ Generalized anxiety disorder, panic disorder, social phobia, obsessive-compulsive disorder and PTSD.

$24 \mathrm{~h}$ of the patient's arrival in the A\&E department. Patients who were admitted to medical or psychiatric beds were seen there. A few were followed up in their own homes - all within 7 days. The research interview was carried out independent from the routine psychiatric assessment. In addition to the SCID, demographic details were obtained, as well as information on previous psychiatric treatment or previous episodes of DSH. All patients also completed the Beck Depression Inventory (BDI), the Beck Hopelessness Inventory (BHI) and the Reasons for Living (RFL) Scale.

In order to make the comparison between the clinical and SCID diagnoses, we obtained patients' charts retrospectively. The chart entry for the relevant A\&E attendance was identified, and the clinical diagnosis was noted. These were originally made and coded using ICD-10 but were transcribed for the purposes of this study to the nearest $D S M-I V$ equivalent. This was agreed between two experienced psychiatrists (CK and CT). Only Axis I diagnoses were dealt with in this study.

Clinical diagnoses were recorded on 154 of the original 167 patients. Seven charts could not be traced, one assessment was incomplete and five were excluded because the primary clinical diagnoses were Axis II and could not be readily compared with the equivalent SCID diagnosis.

At routine clinical assessment, the majority of patients had a single diagnosis recorded. A few were given a secondary diagnosis. In contrast, the SCID generated several diagnoses for most patients (in some cases, five or six diagnoses). These were listed in hierarchical fashion, with the currently most prominent disorder at the top of the list. We compared the single clinical diagnosis with the primary SCID diagnosis. Although this led to the loss of some information, our analysis focused on the most clinically relevant data.

\subsection{Statistical analysis}

All the primary diagnoses made at both routine assessment and structured interview were condensed into 10 broad 
Table 3

Demographic and baseline data

\begin{tabular}{lc}
\hline & $\%(n)$ \\
\hline Age range & \\
$<18$ & $7.8(12)$ \\
$18-30$ & $39.6(61)$ \\
$31-40$ & $21.4(33)$ \\
$41-50$ & $24.7(38)$ \\
$51-60$ & $5.2(8)$ \\
$>60$ & $1.3(2)$ \\
Marital status & \\
Single & $50.0(77)$ \\
Married & $20.7(32)$ \\
Separated/Divorced & $27.9(43)$ \\
Widowed & $1.3(2)$ \\
Previous DSH & \\
History of DSH & $83.8(129)$ \\
1 Prior attempt & $46.7(72)$ \\
2 Prior attempts & $20.7(32)$ \\
$>2$ Attempts & $16.4(25)$ \\
\hline
\end{tabular}

categories (see Table 2) to allow a meaningful statistical comparison. The degree of agreement between the two diagnostic systems was calculated using Cohen's kappa, a statistical measure of correlation. A value of .8 or above is considered to indicate almost perfect agreement, values of $.4-.7$ are moderate to good and .4 and below is fair to poor [2].

Certain subgroups were analyzed further where we thought it likely that there would be diagnostic disagreement. Those diagnosed with adjustment disorder clinically and as depressed by the SCID were compared with those identified as depressed on both diagnostic systems. We expected that the former group would have milder symptoms than the latter. Symptom rating scores were analyzed for these two subgroups using an independent-samples $t$ test. This test was also used to investigate differences in age between the two groups. Chi-squared tests were used to examine possible differences with respect to two other variables - sex and history of previous psychiatric treatment.

\section{Results}

\subsection{Demographic and baseline data}

Demographic data are presented in Table 3 along with information on previous DSH. Patient age ranged from 13 to 77 , with $40 \%$ falling within the $18-30$ bracket; $54.5 \%$ were female and $45.5 \%$ were male; $67.5 \%$ had previous psychiatric treatment either as an outpatient or as an inpatient. A high percentage $(83.8 \%)$ had made at least one previous attempt at DSH.

\subsection{Diagnostic data}

The SCID generated 27 separate diagnoses, while, clinically, 14 were recorded. Ninety-four percent of the patients were diagnosed as having a DSM Axis I disorder on clinical assessment (after transformation from $I C D-10$ ), and the corresponding percentage for the SCID was similar at $93 \%$. The values for each of the diagnostic categories can be seen in Table 2.

There are clear differences in certain areas. Notably, major depressive disorder is diagnosed more commonly by the SCID $(36.4 \%)$ than clinically $(19.5 \%)$. The opposite is true for adjustment disorder - SCID diagnosis 7.8\%; clinical diagnosis $31.8 \%$. Anxiety disorders are identified nine times more often on research assessment (11.7\%) than on clinical assessment (1.3\%). Alcohol- and substanceinduced disorders are also notable areas of disagreement between the two diagnostic systems.

\subsection{Comparison of diagnoses}

The kappa value $(\kappa)$ was .47 , reflecting only a moderate degree of agreement between clinical and standardized diagnoses. The main areas of disagreement are outlined above.

We wished to find out if symptom level influenced how the $\mathrm{A} \& \mathrm{E}$ assessor differentiated between a diagnosis of major depressive disorder and adjustment disorder. The subgroup that was diagnosed clinically with adjustment disorder and by the SCID with major depressive disorder (Group I, $n=17$ ) was compared with the group classed as depressed on both systems (Group II, $n=26$ ) to see if there was any difference between them with respect to symptom severity. A clear difference would suggest that the A\&E assessments were tending to diagnose those with more minor depressive symptoms as having adjustment disorder and identifying as having major depressive disorder those with more severe symptoms. Scores from the BDI, BHI and RFL Scale were examined. A high score on the BDI and BHI indicates a greater degree of depressive symptomatology, whereas on the RFL, higher scores point toward factors protective against depression and suicidal behavior. The mean score on the RFL Scale for Group I was 203, and for Group II, it was 169 . Using the independent-samples $t$ test,

Table 4

Mean scores on rating scales for Group $\mathrm{I}^{\mathrm{a}}$ and Group $\mathrm{II}^{\mathrm{b}}$

\begin{tabular}{|c|c|c|c|c|c|c|}
\hline & \multicolumn{2}{|l|}{ Mean score } & \multirow[t]{2}{*}{ Mean difference } & \multirow[t]{2}{*}{$t$} & \multirow[t]{2}{*}{$P$} & \multirow{2}{*}{$\begin{array}{l}95 \% \text { Confidence } \\
\text { interval of the difference }\end{array}$} \\
\hline & Group I $(n=17)$ & Group II $(n=26)$ & & & & \\
\hline RFL & 203 & 169 & 33.9 & 1.98 & .055 & $-0.71-68.59$ \\
\hline BDI & 25.5 & 29.8 & -4.29 & -1.37 & .177 & $-10.61-2.02$ \\
\hline BHI & 2.47 & 2.81 & -0.34 & -1.02 & .315 & $-1.01-0.33$ \\
\hline
\end{tabular}

${ }^{a}$ Clinical diagnosis - adjustment disorder; SCID diagnosis - major depressive disorder.

${ }^{\mathrm{b}}$ Clinical and SCID diagnoses - major depressive disorder. 
Table 5

BDI scores comparing Group $\mathrm{I}^{\mathrm{a}}(n=17)$ and Group $\mathrm{II}^{\mathrm{b}}(n=26)$

\begin{tabular}{lll}
\hline BDI score & Group I, \% $(n)$ & Group II, \% $(n)$ \\
\hline Severe $(>29)$ & $35(6)$ & $54(14)$ \\
Moderate $(21-29)$ & $35(6)$ & $23(6)$ \\
Mild $(14-20)$ & $30(5)$ & $15(4)$ \\
Minimal $(<14)$ & 0 & $8(2)$ \\
\hline \multicolumn{2}{c}{ a Clinical diagnosis - adjustment disorder; SCID diagnosis - major } \\
depressive disorder. \\
$\quad$ b Clinical and SCID diagnoses — major depressive disorder.
\end{tabular}

there was a trend toward a statistically significant difference ( $t=1.98, P=.055)$. There was no significant difference between the two groups on BDI and BHI scores (see Table 4). There was no difference with respect to age ( $P=.78)$, sex $\left(\chi^{2}=0.34, P=.56\right)$ or past psychiatric history $\left(\chi^{2}=1.60, P=.21\right)$.

We also investigated depressive symptomatology by looking separately at BDI scores by category. Table 5 shows BDI scores for Groups I and II. It would be expected that scores would be higher in the group classed as depressed by both systems (Group II) and lower in the major depressive disorder/adjustment subgroup (Group I). Upon looking at the results, the former group appears to have a greater proportion with higher scores. However, this did not reach statistical significance. The presence of two patients classed as depressed on the SCID who also had minimal BDI scores appears to be contradictory.

It was notable that four anxiety-type disorders were diagnosed by the SCID, but they did not appear as clinical diagnoses. These were generalized anxiety disorder, obsessive-compulsive disorder, social phobia and panic disorder, representing eight cases. Posttraumatic stress disorder (PTSD; also included in the anxiety group) was diagnosed on 10 occasions by the SCID and only twice clinically.

\section{Discussion}

Our findings show moderate agreement between routine A\&E assessment and a research instrument. A high standard was set in the analysis by expecting the single clinical diagnosis to correlate with the primary SCID diagnosis, rather than correspond to any of the SCID diagnoses listed for each case. Therefore, in some ways, it is not surprising that the kappa value was not higher than .47. However, other reasons why the level of diagnostic agreement is only moderate need to be addressed. Previous evidence shows that subsequent aftercare is influenced by the diagnosis made at psychiatric evaluation in A\&E [3], and therefore, it is important that a degree of accuracy is achieved. Clearly, one reason could be an underdiagnosis of certain disorders at A\&E evaluation. Areas of diagnostic difficulty need to be considered also.

\subsection{Previous studies}

We found that on clinical diagnosis, the three most common disorders were adjustment disorder, major depres- sive disorder and alcohol misuse/dependence. Other recent studies have examined the diagnostic breakdown of similar populations of patients, with somewhat varied results. In a sample of DSH patients admitted to an Irish general hospital [4], adjustment disorder was found to be the most frequent clinically made diagnosis, followed by major depressive disorder and alcohol dependence. In contrast, another study of a similar group identified major depressive disorder as the most frequent clinical diagnosis, with a low incidence of adjustment disorder [5]. In the same study, anxiety disorders were identified in $14 \%$ of the patients. Most studies agree that alcohol misuse/dependence is common in this population, but disorders comorbid with alcohol (especially major depressive disorder) are often ignored [6].

Several previous studies have compared accuracy of clinical diagnosis at emergency assessment with diagnosis at subsequent discharge on patients who required psychiatric admission. Two studies $[7,8]$ used a kappa calculation and concluded that overall levels of agreement were acceptable for the emergency assessment situation. Both studies used clinician-made diagnoses as their standard for comparison. We are aware of only one other study that uses research criteria to measure against clinical diagnosis [1]. In a similar finding to ours, major depressive disorder and alcohol misuse/dependence were diagnosed less frequently on clinical assessment (adjustment disorder was not included as a separate diagnosis). Comorbidity was not well recognized. The authors conclude that this points to an underdiagnosis of certain disorders, which could be improved on by using more well defined diagnostic criteria.

\subsection{Diagnostic difficulties}

\subsubsection{Major depressive disorder and adjustment disorder}

Our results show a clear disagreement in the area of major depressive disorder and adjustment disorder, with many of those classed as depressed by the SCID having a clinical diagnosis of adjustment disorder.

In an important study in 1979, Urwin and Gibbons [9] looked at a sample of patients who deliberately self-poison and hypothesized that only a small number would be classed as psychiatric "cases." In fact, a larger number than expected were diagnosed as depressed, but many of these were found to be of "minor severity." The authors identified this minor severity group as problematic, as it was doubtful if they would benefit from treatment. More recently, Casey et al. [10] comment on the "overelastic" use of the term depression, at the expense of adjustment disorder. This borderline between depressive disorder as a pathological entity and understandable emotional distress in response to a difficult situation has always been problematic and is particularly relevant in large urban A\&E departments where social and situational problems are common.

We therefore expected to find that those diagnosed as having adjustment disorder clinically and as depressed by the SCID would show evidence of milder pathology than 
those identified as depressed on both diagnostic systems. Our results suggested a difference with respect to scores on the RFL Scale, although the significance was borderline. The other scales and demographic variables did not suggest a clear difference, but the numbers in this part of the analysis were small.

The use of diagnostic criteria gives some guidance on these problems but has potential limitations. Some authors $[11,12]$ advocate the use of structured questionnaires in the assessment of suicidal behavior. However, overrigidity in diagnosis could be a result. Casey takes the view that clinical judgement in diagnosis should be emphasized, rather than an overreliance on rigid diagnostic criteria. In an earlier study into the applicability of $I C D-10$ to diagnosis in liaison psychiatry, the authors found that the usefulness of diagnostic criteria was limited when dealing with "the blurred area between adjustment disorder and depression" [13]. Our results bear out this tension between the practicalities of everyday clinical assessment and the stringency of research criteria.

\subsubsection{Anxiety disorders}

A less-expected area of disagreement was in the diagnosis of anxiety disorders. This group included generalized anxiety disorder, panic disorder, social phobia, obsessive-compulsive disorder and PTSD. None of these disorders was identified clinically, apart from two cases of PTSD. We concluded that these disorders are underdiagnosed at A\&E psychiatric evaluation. One possible reason is that some cases of anxiety are not considered as a "severe mental illness" and may not be referred for secondary care intervention. They may therefore be overlooked by the assessing professional in the A\&E department. However, they are amenable to treatment whether in a primary or secondary care setting and should not be ignored. Another explanation for this low identification is that they are often comorbid with other conditions that may take precedence in diagnosis, thus making it seem that the anxiety disorder has been missed, when in fact this may not be the case. Anxiety can often coexist with other problems such as depression and substance misuse, and in these cases, the diagnostic distinction can be difficult to make.

We would have expected better recognition of PTSD in Northern Ireland. Many patients who present with posttraumatic symptoms are related to incidents of sectarian or paramilitary violence [14]. Despite the cease-fires in the 1990s and some limited political progress in recent years, paramilitary activity has been ongoing and its victims continue to present at A\&E departments. In view of this, it is surprising that our recognition of PTSD is so poor. However, with regard to other populations, these local issues may mean that this particular finding is less generalizable overall.

\subsubsection{Alcohol misuse/dependence}

Alcohol misuse/dependence is another area that shows disparity. The SCID identified $20 \%$ as having a primary alcohol problem, while the value for clinical assessment was higher at $30 \%$. Of those diagnosed clinically but not by the SCID, approximately half have major depressive disorder as the SCID diagnosis. Other studies have shown that rates of comorbid depressive disorder and alcohol misuse/dependence are high. Haw et al. [6] found that in a group of DSH patients who abused or were dependent on alcohol, $75 \%$ had comorbid depression. Our findings suggest that we are identifying patients who have alcohol misuse/dependence, but we may be underestimating the presence of other disorders. It can be difficult when assessing mood and alcohol intake to separate out cause and effect and to judge which is the primary disorder. This can be especially difficult at A\&E assessment where time and information may be limited. In an area where diagnostic difficulties already exist, alcohol is clearly a further complicating factor.

\section{Clinical implications and limitations}

We found that major depressive disorder, adjustment disorder and alcohol misuse/dependence are commonly diagnosed in A\&E settings on both clinical and research assessments.

However, there is a disparity between these two diagnostic systems in the identification of major depressive disorder and adjustment disorder. This not only suggests a possible clinical underdiagnosis of depression in the A\&E setting but also highlights a problematic lack of clarity in diagnostic boundaries. Anxiety disorders are diagnosed much more frequently using the research tool, pointing to a clear underdiagnosis at psychiatric assessment in A\&E settings.

There is no information on the patients who did not consent to take part. As a group, they may have differed from the study sample, but this cannot be assessed. The numbers in the analysis are relatively small, and the power to detect differences between subgroups is limited for this reason. The use of only the primary diagnoses in the kappa comparison may have resulted in the loss of some information, particularly regarding comorbidity. Finally, the omission of Axis II diagnoses is a weakness. As with alcohol, the presence of comorbid personality disorder adds complexity to the clinical picture and worsens prognosis. The inclusion of this information would be interesting, but unfortunately, it is beyond the scope of this study.

\section{References}

[1] Suominen K, Isometsa E, Henriksson M, Suokas J, Ostamo A Lonnqvist J. Consultation versus research diagnosis of mental disorders among suicide attempters. Nord J Psychiatry 1999;53: 253-6.

[2] Fayers P, MacHin D. Quality of life: assessment, analysis and interpretation. Chichester (UK): Wiley; 2000. p. 59.

[3] Khuri R, Wood K. The role of diagnosis in a psychiatric emergency setting. Hosp Community Psychiatry 1984;35:715-8.

[4] McCauley M, Russell V, Bedford D, Khan A, Kelly R. Assessment following deliberate self-harm: who are we seeing and are we following guidelines? Ir J Psychol Med 2001;18:116-9. 
[5] Haw C, Hawton K, Houston K, Townsend E. Psychiatric and personality disorders in deliberate self-harm patients. $\mathrm{Br} \mathrm{J}$ Psychiatry 2001;178:48-54.

[6] Haw C, Houston K, Townsend E, Hawton K. Deliberate self-harm patients with alcohol disorders: characteristics, treatment, and outcome. Crisis 2001;22:93-101.

[7] Lieberman PB, Baker FM. The reliability of psychiatric diagnosis in the emergency room. Hosp Community Psychiatry 1985;36:291-3.

[8] Warner MD, Peabody CA. Reliability of diagnosis made by psychiatric residents in a general emergency department. Psychiatr Serv 1995;46:1284-7.

[9] Urwin P, Gibbons JL. Psychiatric diagnosis in self-poisoning patients. Psychol Med 1979;9:501-7.
[10] Casey P, Dowrick C, Wilkinson G. Adjustment disorders. Br J Psychiatry 2001;179:479-81.

[11] Malone KM, Szanto K, Corbitt EM, Mann J. Clinical assessment versus research methods in the assessment of suicidal behaviour. Am J Psychiatry 1995;152:1601-7.

[12] Helzer JE, Clayton PJ, Pambakian R, Woodruff A. Concurrent diagnostic validity of a structured psychiatric interview. Arch Gen Psychiatry 1978;35:849-53.

[13] Dauncey MK, Cooper JE. Diagnostic problems in liaison psychiatry and the ICD-10. J Psychosom Res 1985;34:287-94.

[14] Daly O. Northern Ireland: the victims. Br J Psychiatry 1999;175: $201-4$. 